\title{
Possible Association of Amelogenin to High Caries Experience in a Guatemalan-Mayan Population
}

\author{
K. Deeley ${ }^{a} \quad$ A. Letra ${ }^{a} \quad$ E.K. Rose ${ }^{a} \quad$ C.A. Brandon ${ }^{a} \quad$ J.M. Resick ${ }^{a} \quad$ M.L. Marazita ${ }^{a}$ c-e \\ A.R. Vieira ${ }^{a-d}$ \\ Departments of a Oral Biology and ${ }^{b}$ Pediatric Dentistry, and ${ }^{c}$ Center for Craniofacial and Dental Genetics, School of \\ Dental Medicine, d Department of Human Genetics, Graduate School of Public Health, and e Department of Psychiatry, \\ School of Medicine, University of Pittsburgh, Pittsburgh, Pa., USA
}

\section{Key Words}

Ameloblastin $(A M B N) \cdot$ Amelogenin $(A M E L X) \cdot$ Enamelin

(ENAM) • Tuftelin-1 (TUFT1) • Tuftelin interacting protein 11

(TFIP11)

\begin{abstract}
There is evidence for a genetic component in caries susceptibility, but the disease is greatly influenced by environmental factors, which are extremely difficult to control in humans. For the present study, we used DNA samples collected from 110 unrelated, non-cleft individuals older than 12 years of age from Tiquisate, Guatemala: a population with similar cultural, dietary and hygiene habits, similar access to the dentist and fluoride exposure. Forty-four individuals were designated 'very low caries experience' (DMFT $\leq 2)$, and 66 were designated 'higher caries experience' (DMFT $\geq 3$ ). Single-nucleotide polymorphism markers were genotyped in selected candidate genes (ameloblastin, amelogenin, enamelin, tuftelin-1, and tuftelin interacting protein 11) that influence enamel formation. Having at least one copy of the rare amelogenin marker allele was associated with increased ageadjusted caries experience. This association was stronger in individuals with higher DMFT (DMFT $\geq 20 ; p=0.0000001$ ). Our results suggest that variation in amelogenin may contribute to caries susceptibility in the population studied. The
\end{abstract}

approach of comparing individuals with extremely distinct caries experiences could be valuable for decreasing the potential influence of environmental factors on genetic studies of caries.

Copyright $\odot 2007$ S. Karger AG, Basel

Studies of twins [reviewed by Shuler, 2001], families [Klein and Palmer, 1940; Klein, 1946], and animal breeding [Hunt et al., 1944; Rosen et al., 1961] have indicated that caries has a genetic component. Studies on monozygotic twin pairs reared apart suggest a genetic contribution to caries of $40 \%$ [Conry et al., 1993]. Studies of twins reared together estimated the heritability for caries, adjusted for age and gender, as 45-64\% [Bretz et al., 2005]. However, shared behavior, practice, and habits within families can be expected to contribute to covariance between relatives and to mimic genetic correlation if not controlled by the experimental design [Potter, 1990]. This is particularly true for caries, which can be profoundly affected by dietary sugar intake and/or oral hygiene practices within families.

The $\mathrm{H}-2$ region on chromosome 17 was linked to susceptibility to caries in mice [Suzuki et al., 1998], as was chromosome 2 [Uematsu et al., 2003]. A genome-wide scan performed in genetic crosses of $\mathrm{C} 3 \mathrm{H} / \mathrm{HeJ}$ (caries- 
resistant) and C57BL/6J (caries-susceptible) mice inoculated with Streptococcus mutans serotype c detected three suggestive quantitative trait loci (QTL) on chromosomes 1,2 , and 7, one significant QTL on chromosome 2, and one highly significant QTL on chromosome 8 [Nariyama et al., 2004].

Only recently has a genetics study in human caries been reported. Tuftelin-1 genotypes appeared to interact with levels of $S$. mutans infection in children with early childhood caries [Slayton et al., 2005].

In the current study we compared individuals living in the same area and with similar cultural backgrounds and access to dental care in an attempt to reduce the influence of environmental confounders. Individuals with very low caries experience were compared with individuals with higher caries experience. Genetic markers in genes involved in enamel formation were tested to assess the possible association between such gene variants and caries experience.

\section{Subjects and Methods}

This project is part of a larger research program on factors that contribute to development of cleft lip and palate and other craniofacial anomalies and oral conditions. The study was done in collaboration with the nonprofit organization Children of the Americas. Between January 15 and January 19, 2006, individuals and their family members seeking cleft lip and palate repair at the mission base hospital in Pueblo Nuevo Tiquisate, Guatemala, were invited to participate in our study. This project was approved by both the University of Pittsburgh Institutional Review Board and the Oversight Ethics Committee of the Hospital Nacional de Tiquisate, and all subjects gave informed, written consent. Ageappropriate assent documents were used for children between 7 and 14 years of age and informed, written consent was obtained from the child, as well as from the parents.

After more than 30 years of civil war, a more peaceful time started in Guatemala in 1996 but 55\% of the population lives on less than USD 1 per day and $90 \%$ of the indigenous population lives below the poverty line. Pueblo Nuevo Tiquisate, population 16,801 inhabitants [Instituto Nacional de Estadistica Guatemala, 2002], is located in the Escuintla department, central Guatemala. The population is primarily rural.

We recruited 217 individuals for our studies. All individuals received an oral and dental examination performed by a single examiner (A.R.V.). DMFT was scored according to World Health Organization guidelines. Most individuals reported brushing their teeth at least once a day with a toothbrush and toothpaste. Drinking water in the region is not artificially fluoridated. Saliva samples were obtained from all participants and stored in Oragene DNA Self-Collection kits (DNA Genotek Inc.). DNA was extracted according to the manufacturer's instructions. For the genetics analysis, 110 unrelated individuals were classified as either 'very low caries experience' (DMFT $\leq 2 ; \mathrm{n}=44$ ) or as 'higher caries experience' (DMFT $\geq 3 ; \mathrm{n}=66$ ). In instances where related
Table 1. Demographic characteristics of the sample

\begin{tabular}{|c|c|c|}
\hline Characteristics & $\begin{array}{l}\text { Very low caries } \\
\text { experience }(\mathrm{DMFT} \leq 2)\end{array}$ & $\begin{array}{l}\text { Higher caries } \\
\text { experience }(\mathrm{DMFT} \geq 3)\end{array}$ \\
\hline Number & 44 & 66 \\
\hline Males & 20 & 25 \\
\hline Females & 26 & 39 \\
\hline \multicolumn{3}{|c|}{$\chi^{2}=0.216 ; p=0.64$} \\
\hline Age (mean $\pm \mathrm{SD})$ & $23.5 \pm 5.21$ & $32.62 \pm 11.77$ \\
\hline Age range & $14-42$ & $16-60$ \\
\hline \multicolumn{3}{|c|}{ t test $; \mathrm{p}<0.001$} \\
\hline \multicolumn{3}{|l|}{ DMFT scores } \\
\hline 0 & 26 & - \\
\hline 1 & 8 & - \\
\hline 2 & 10 & - \\
\hline 3 & - & 6 \\
\hline 4 & - & 4 \\
\hline 5 & - & 3 \\
\hline 6 & - & 6 \\
\hline 7 & - & 4 \\
\hline 8 & - & 3 \\
\hline 9 & - & 5 \\
\hline 10 & - & 2 \\
\hline 11 & - & 3 \\
\hline 12 & - & 4 \\
\hline 13 & - & 2 \\
\hline 14 & - & 5 \\
\hline 15 & - & 2 \\
\hline 16 & - & 3 \\
\hline 17 & - & 5 \\
\hline 19 & - & 2 \\
\hline 20 & - & 3 \\
\hline 21 & - & 3 \\
\hline 27 & - & 1 \\
\hline
\end{tabular}

individuals were selected, only the oldest individual belonging to the family was included in the analysis. Since there are reports that suggest that cleft individuals have a higher incidence of caries, individuals presenting an oral cleft were not considered for these analyses, even though these reports are not confirmed by others [reviewed by Cheng et al., 2007]. Only individuals 12 years of age or older were included. Demographic characteristics of the two groups are presented in table 1.

Power calculations [Purcell et al., 2003], assuming that our marker alleles were in complete linkage disequilibrium with the genetic variant contributing to caries susceptibility, and that the chance of having a distinct (very low or very high) caries susceptibility increased fivefold when having one copy of the caries susceptibility allele, suggested a $78 \%$ power to detect a possible association with our sample size. This procedure was for a marker $\mathrm{B}$ in linkage disequilibrium with our test locus A. Other parameters specified in the calculations were the high-risk allele frequency for the allele $A$ (set at 0.1); the disease prevalence in the general population [set at 0.1 , corresponding to the approximate frequency of caries-free individuals or highly affected individuals 


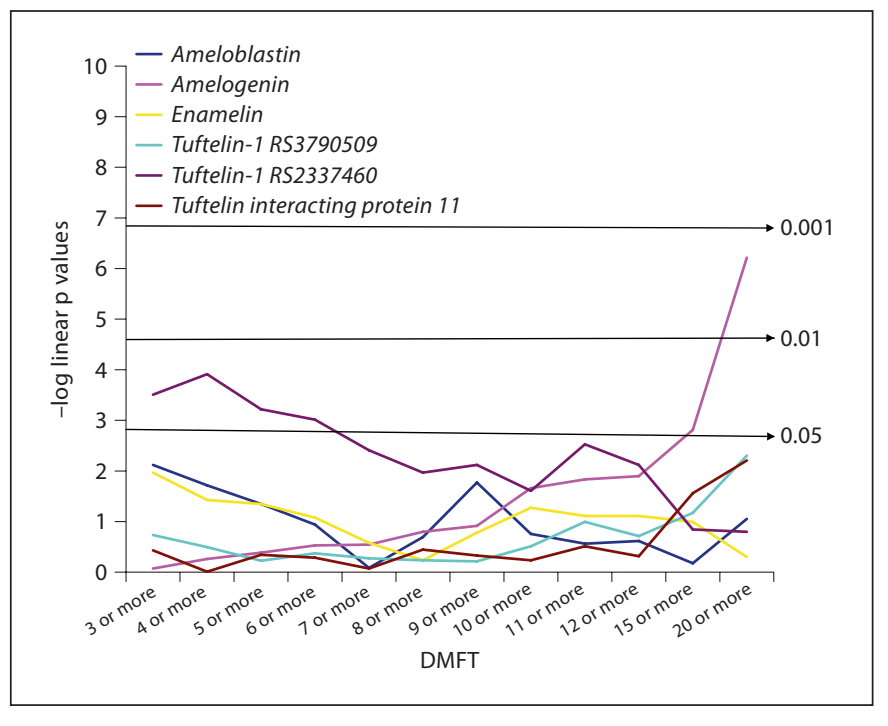

Fig. 1. Linear regression results comparing individuals with low caries experience (DMFT scores up to 2) versus individuals with increasing caries experience. - - log linear $\mathrm{p}$ values plotted against DMFT cutoff. The tuftelin-1 rs2337360 marker showed significant $\mathrm{p}$ values for DMFT $\geq 3$ up to $\geq 6$. The amelogenin marker studied showed borderline significant results for DMFT $\geq 15$ and highly significant results for DMFT $\geq 20$.

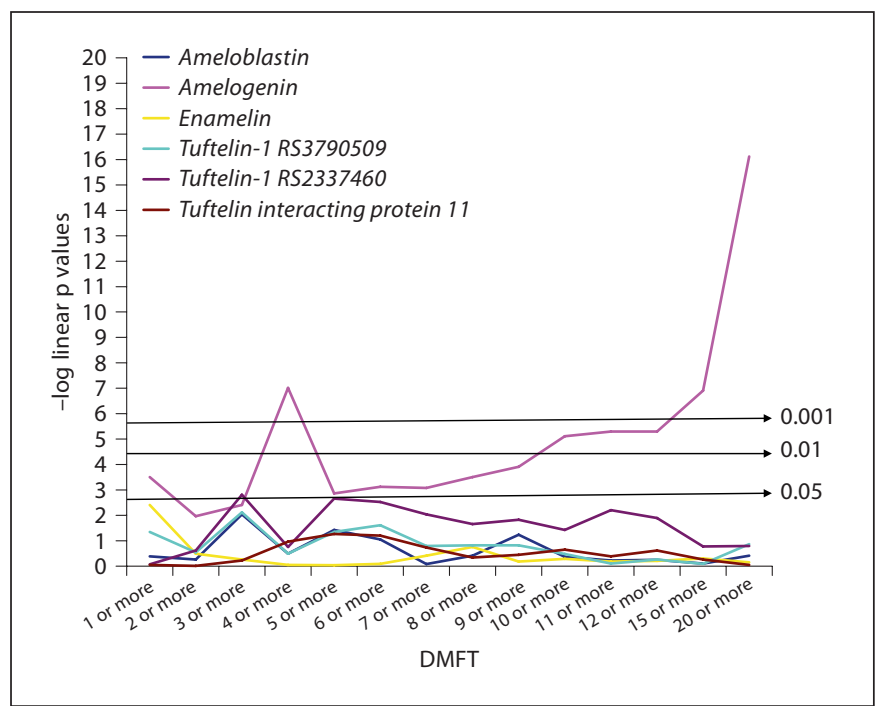

Fig. 2. Linear regression results comparing caries-free individuals versus individuals with increasing caries experience. -log linear $\mathrm{p}$ values plotted against DMFT cutoff. The amelogenin marker studied consistently showed significant/borderline results for DMFT $\geq 4$ with highly significant results from DMFT $\geq 10$ to $\mathrm{DMFT} \geq 20$.

Table 2. Candidate gene markers studied

\begin{tabular}{lllll}
\hline Gene & Locus & Marker public ID & Base pair change & $\begin{array}{l}\text { Minor allele frequency (observed } \\
\text { in the studied population) }\end{array}$ \\
\hline Ameloblastin (AMBN) & $4 \mathrm{q} 21$ & hCV496502 & $\mathrm{G} \rightarrow \mathrm{T}$ & 0.22 \\
Amelogenin (AMELX) & $\mathrm{Xp} 22.31-\mathrm{p} 22.1$ & $\mathrm{hCV} 2190967$ & $\mathrm{C} \rightarrow \mathrm{T}$ & 0.06 \\
Enamelin (ENAM) & $4 \mathrm{q} 13.3$ & $\mathrm{rs} 3796704$ & $\mathrm{~A} \rightarrow \mathrm{G}$ & 0.10 \\
Tuftelin-1 (TUFT1) & $1 \mathrm{q} 21$ & $\mathrm{rs} 3790506$ & $\mathrm{~A} \rightarrow \mathrm{G}$ & 0.41 \\
Tuftelin interacting protein 11 (TFIP11) & 22q12.1 & rs2337360 & $\mathrm{G} \rightarrow \mathrm{A}$ & 0.25 \\
\hline
\end{tabular}

(DMFT >20)], and the genotype risks for the Aa and AA genotypes relative to the baseline aa genotype risk.

Genotyping was carried out using TaqMan chemistry [Ranade et al., 2001] and performed on an Applied Biosystems 7900 HT Sequence Detection System machine. Single-nucleotide polymorphism markers were selected within candidate genes involved in enamel formation (table 2).

Student $t$ and $\chi^{2}$ tests were performed to compare gender and age frequencies and to test for deviations in the genotype distributions from Hardy-Weinberg equilibrium. Because there was a statistically significant difference between the mean ages of the two comparison groups (table 1), we performed linear regression to account for the age difference using the EpiInfo 3.3.2 software (http://www/cdc/gov/epiinfo). Genotype data were included in the model and, based on the observed genotype frequencies of the markers, individuals with one or two copies of the rare alleles were combined together. In the first round of analysis, individuals with low caries experience were compared to individuals with higher DMFT scores. Then all analyses were repeated using caries-free individuals as a comparison group (individuals with DMFT 1 or 2 excluded). Statistical significance was determined at $\alpha=0.05$.

\section{Results}

All genotypes were in Hardy-Weinberg equilibrium. Figures 1 and 2 summarize the results of the linear regression analysis. Figure 1 shows the -log linear p values 
of the comparisons between low caries experience individuals and individuals with higher caries experience. Amelogenin showed significant association with very high levels of caries experience (DMFT $\geq 15, p=0.06$; $\geq 20, p=0.002$ ). When caries-free individuals were used as comparison group (fig. 2), amelogenin showed associations with increasing DMFT scores and the association was stronger when the number of affected teeth was very high $(\mathrm{DMFT} \geq 1, \mathrm{p}=0.03 ; \geq 2, \mathrm{p}=0.14 ; \geq 3, \mathrm{p}=0.09 ; \geq 4$, $\mathrm{p}=0.0009 ; \geq 5, \mathrm{p}=0.057 ; \geq 6, \mathrm{p}=0.044 ; \geq 7, \mathrm{p}=0.046 ;$ $\geq 8, \mathrm{p}=0.03 ; \geq 9, \mathrm{p}=0.02 ; \geq 10, \mathrm{p}=0.006 ; \geq 11, \mathrm{p}=0.005 ;$ $\geq 12, \mathrm{p}=0.005 ; \geq 15, \mathrm{p}=0.001 ; \geq 20, \mathrm{p}=0.0000001$ ).

The tuftelin-1 rs2337360 marker showed significant association when individuals with DMFT $\leq 2$ were compared with individuals with DMFT $\geq 3(\mathrm{p}=0.03), \geq 4$ $(\mathrm{p}=0.02), \geq 5(\mathrm{p}=0.042)$, and $\geq 6(\mathrm{p}=0.049)$, but not when caries-free individuals were used in the comparisons. All other marker genotypes were not statistically associated with DMFT scores.

\section{Discussion}

To decrease heterogeneity, individuals with distinct caries experiences were compared. All individuals that participated in this study had similar access to oral care and shared a set of cultural norms related to dietary and oral hygiene habits. In addition, their fluoride exposure is likely to have been very similar, with no artificially fluoridated drinking water. In view of the heterogeneity of age in our samples, and the importance of age as a confounder, we took age into consideration when analyzing the genotyping data.

This is the first report to suggest that genetic variation in the amelogenin gene contributes to susceptibility to caries. Previous work [Slayton et al., 2005] showed that association between genetic variation of tuftelin-1 and caries could only be detected when the interaction with $S$. mutans levels was included in the model. We did find some evidence for an association between the tuftelin-1 rs2337360 marker and higher caries experience, but these results were not consistent across all the DMFT cutoffs. In addition, the other tuftelin-1 marker did not show evidence for an association, which could be due to decreased sample sizes of the higher DMFT score groups. Also, we did not have any microbiological data and it is possible that tuftelin-1 contribution to susceptibility to caries may be more clearly detected when $S$. mutans levels are considered during analysis. Slayton et al. [2005] did not find an association between amelogenin markers and caries experience in their study. However, our study and that of Slayton et al. [2005] differ in populations (Iowa, USA versus Guatemala), age groups (3-5-year-old children versus individuals 12 or older), caries experience $(61.1 \%$ cariesfree in Iowa versus $23.6 \%$ in Tiquisate) and caries measurement (DMFS including white spot lesions versus traditional DMFT).

Previous animal models [Suzuki et al., 1998; Uematsu et al., 2003; Nariyama et al., 2004] suggested that mouse chromosomes 1, 2, 7, 8, and 17 could harbor a caries susceptibility gene but, for technical reasons, these studies did not investigate sex chromosomes. In consequence, amelogenin (located in the X chromosome) was not investigated.

The main disadvantage of using a case-control design for detecting an association between a genetic marker and the phenotypic trait is that power declines rapidly with a decreasing degree of linkage disequilibrium between the trait locus and the candidate locus [Sham et al., 2000]. Since linkage disequilibrium is influenced by numerous factors, such as population size and structure, migration, and selection, the reduction of linkage disequilibrium with increasing recombination fraction is less rapid in a population with a more recent admixture effect (such as the population studied, which basically comprises the combination of Amerindians and Spaniards that happened about ten generations ago). Our power calculations indicate that our approach of investigating separately individuals with dramatic differences in caries experience likely further improved our chances of detecting an association.

Even with stricter criteria for significance (e.g. Bonferroni correction), the association between high caries experience (DMFT $\geq 20 ; \mathrm{p}=0.0000001)$ and variation in amelogenin would remain statistically significant. Testing 6 markers and 14 DMFT score groups (fig. 2) would lower alpha from 0.05 to $0.0005952(0.05 / 84)$. The adjustment for multiple testing in combination with the consistent association between increasing DMFT scores and the amelogenin marker suggests that the results are not due to type I error. Although multiple testing is always an issue to be considered when multiple genetic markers are being tested, the likely small contribution of each individual gene to any complex trait (including caries) indicates that caution has to be exercised to not increase type II errors, and consequently ignore possible true associations that may explain a small percentage of cases.

Amelogenins are highly conserved proteins and constitute $90 \%$ of the enamel organic matrix. Mutations in amelogenin lead to X-linked amelogenesis imperfecta 
[Wright, 2006]. Our results suggest that genetic variation in amelogenin contributes to higher susceptibility to caries. One can argue that mildly defective amelogenin or slightly smaller amounts of this protein could lead to some degree of disorganization of the enamel prisms that increases the individual's susceptibility to caries.

A susceptibility gene for caries in the human X chromosome raises interesting possibilities. There is a nearly universal female gender bias in caries [Lukacs and Largaespada, 2006]. Higher caries prevalence among females is often explained by early eruption of teeth, snacking during food preparation, pregnancy and hormonal influences. A contribution of amelogenin to caries susceptibility may shed some light on gender differences in caries prevalence. The amelogenin results were driven by 7 women with one copy of the rare amelogenin marker allele (data not shown): 3 with DMFT score of 1 and the others with DMFT scores $6,15,17$, and 20. This would argue in favor of an X-linked dominant disease model with variable expressivity (although an X-linked recessive model cannot be excluded because no males carried the amelogenin risk allele in the population studied). When the analysis was carried out with caries-free individuals in the comparison group (when the 3 women with DMFT scores of 1 were not included), the association between amelogenin and DMFT was stronger. Therefore, another possibility is the presence of nonrandom $\mathrm{X}$-inactivation patterns contributing to caries susceptibility. Normal females are mosaic of two cell populations, each expressing the alleles from one X chromosome or the other. Thus, in female carriers of an X-linked mutation, approximately $50 \%$ of cells on average have the normal allele on the active $\mathrm{X}$ chromosome (with the mutant allele being on the inactive chromosome), and these functionally normal cells can spare females from clinical effects of an X-linked disease [Puck and Willard, 1998]. Occasionally, however, females do have clinical manifestations of an X-linked disorder due to skewed X-inactivation patterns (more than $50 \%$ of the active chromosomes carry the mutant allele). It is possible that the 4 females with higher caries experience had a preferential inactivation of the $\mathrm{X}$ chromosome that did not carry the high caries susceptibility amelogenin variant and therefore had higher caries experience. On the other hand, the 3 females with DMFT scores equal 1 would have a closer to 1:1 ratio of chromosomes with normal and defective amelogenin alleles. However, this epigenetic mechanism does not imply that males would have a lower risk for developing caries and does not explain the higher prevalence of caries in females seen in epidemiological studies.
The Y chromosome has an amelogenin-like gene (AMELY) that corresponds to the amelogenin gene in the X chromosome (AMELX) [Nakahori et al., 1991]. Both $A M E L X$ and $A M E L Y$ genes are transcriptionally active in male developing tooth buds [Salido et al., 1992]. These are not pseudoautosomal genes, since females heterozygous for mutations in the AMELX gene causing amelogenesis imperfecta show nonrandom $\mathrm{X}$ inactivation and a mosaic pattern of enamel abnormality. In addition, the promoting effect of $A M E L Y$ on tooth growth seems more effective than that of AMELX [Alvesalo and Portin, 1980]. Therefore, it is possible that the presence of an AMELX caries susceptibility allele in males may be compensated by the homologous (and of course active) AMELY gene.

The 7 women with variation in amelogenin reported that they had similar oral hygiene habits and access to dental care. Since in the majority of individuals most of the caries experience is manifested by the age of 12 , and all 7 women were 19-28 years old, other factors, such as dietary habits combined with distinct bacterial colonization, could have played a role in the caries susceptibility of these 7 women during their childhood. Future study designs based on families might help to better understand the relationship between genetic and environmental factors in caries susceptibility.

In summary, our results suggest that variation in the amelogenin gene may be a factor in caries susceptibility. The approach of comparing individuals with extremely distinct caries experiences might be valuable for decreasing the potential influence of environmental factors on genetic studies of caries.

\section{Acknowledgments}

The authors would like to thank the individuals who participated in this study. Also, we are indebted to Children of the Americas, Inc., for its support during the 2006 medical mission. We thank Dr. Steve Wendell and Monica Varela for technical support. This research is supported by NIH/NIDCR Grants R01-DE016148, P50-DE016215, R21-DE016930 and R21-DE014899.

References

Alvesalo L, Portin P: 47,XXY males: sex chromosomes and tooth size. Am J Hum Genet 1980; 32:955-959.

Bretz WA, Corby PM, Schork NJ, Robinson MT, Coelho M, Costa S, Melo Filho MR, Weyant RJ, Hart TC: Longitudinal analysis of heritability for dental caries traits. J Dent Res 2005; 84:1047-1051. 
Cheng LL, Moor SL, Ho CTC: Predisposing factors to dental caries in children with cleft lip and palate: a review and strategies for early prevention. Cleft Palate Craniofac J 2007;44: 67-72.

-Conry JP, Messer LB, Boraas JC, Aeppli DP, Bouchard TJ Jr: Dental caries and treatment characteristics in human twins reared apart. Arch Oral Biol 1993;38:937-943.

Hunt HR, Hoppert CA, Erwin WG: Inheritance of susceptibility to caries in albino rats (Mus norvegicus). J Dent Res 1944;23:385-401.

Instituto Nacional de Estadística Guatemala. National Census 2002.

Klein H: The family and dental disease. IV. Dental disease (DMF) experiences in parents and offspring. J Am Dent Assoc 1946;33:735743.

-Klein H, Palmer CE: Dental caries in brothers and sisters of immune and susceptible children. Milbank Mem Fund Q 1940;18:67-82.

Lukacs JR, Largaespada LL: Explaining sex differences in dental caries prevalence: saliva, hormones, and 'life-history' etiologies. Am J Hum Biol 2006;18:540-555.

- Nakahori Y, Takenaka O, Nakagome Y: A human $\mathrm{X}-\mathrm{Y}$ homologous region encodes 'amelogenin’. Genomics 1991;9:264-269.
- Nariyama M, Shimizu K, Uematsu T, Maeda T: Identification of chromosomes associated with dental caries susceptibility using quantitative trait locus analysis in mice. Caries Res 2004;38:79-84.

Potter RH: Twin half-sibs: a research design for genetic epidemiology of common dental disorders. J Dent Res 1990;69:1527-1530.

Puck JM, Willard HF: X inactivation in females with X-linked disease. N Engl J Med 1998; 338:325-328.

Purcell S, Cherny SS, Sham PC: Genetic power calculator: design of linkage and association genetic mapping studies of complex traits. Bioinformatics 2003;19:149-150.

Ranade K, Chang MS, Ting CT, Pei D, Hsiao CF, Olivier M, Pesich R, Hebert J, Chen YD, Dzau VJ, Curb D, Olshen R, Risch N, Cox DR, Botstein D: High-throughput genotyping with single nucleotide polymorphisms. Genome Res 2001;11:1262-1268.

Rosen S, Hunt HR, Hoppert CA: Hereditary limitations of the infectious and transmissible nature of experimental dental caries. Arch Oral Biol 1961;5:92-97.
Salido EC, Yen PH, Koprivnikar K, Yu LC, Shapiro LJ: The human enamel protein gene amelogenin is expressed from both the $\mathrm{X}$ and the Y chromosomes. Am J Hum Genet 1992; 50:303-316.

Sham PC, Cherny SS, Purcell S, Hewitt JK: Power of linkage versus association analysis of quantitative traits, by use of variance-components models, for sibship data. Am J Hum Genet 2000;66:1616-1630.

Shuler CF: Inherited risks for susceptibility to dental caries. J Dent Educ 2001;65:10381045

Slayton RL, Cooper ME, Marazita ML: Tuftelin, mutans streptococci, and dental caries susceptibility. J Dent Res 2005;84:711-714.

Suzuki N, Kurihara Y, Kurihara Y: Dental caries susceptibility in mice is closely linked to the $\mathrm{H}-2$ region on chromosome 17. Caries Res 1998;32:262-265.

Uematsu T, Nariyama M, Shimizu K, Maeda T: Mapping of affected gene(s) to dental caries susceptibility on mouse chromosome 2. Pediatr Dent J 2003;13:75-81.

Wright JT: The molecular etiologies and associated phenotypes of amelogenesis imperfecta. Am J Med Genet A 2006;140:2547-2555. 